\title{
Rapid development of an inactivated vaccine candidate for SARS-CoV-2
}

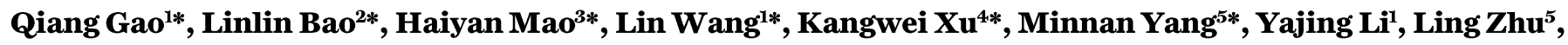
Nan Wang ${ }^{5}$, Zhe Lv5, Hong Gao ${ }^{2}$, Xiaoqin Ge', Biao Kan ${ }^{6}$, Yaling Hu', Jiangning Liu'², Fang Cai', Deyu Jiang', Yanhui Yin', Chengfeng Qin ${ }^{7}$, Jing Li', Xuejie Gong1, Xiuyu Lou ${ }^{3}$, Wen Shi ${ }^{3}$, Dongdong Wu', Hengming Zhang',

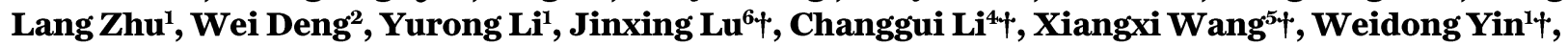
Yanjun Zhang ${ }^{3}+$, Chuan Qin ${ }^{2} \dagger$

${ }^{1}$ Sinovac Biotech Ltd., Beijing, China. ${ }^{2}$ Key Laboratory of Human Disease Comparative Medicine, Chinese Ministry of Health, Beijing Key Laboratory for Animal Models of Emerging and Remerging Infectious Diseases, Institute of Laboratory Animal Science, Chinese Academy of Medical Sciences and Comparative Medicine Center, Peking Union Medical College, Beijing, China. ${ }^{3}$ Department of Microbiology, Zhejiang Provincial Center for Disease Control and Prevention, Hangzhou, China. ${ }^{4}$ Division of Respiratory Virus Vaccines, National Institute for Food and Drug Control, Beijing, China. ${ }^{5}$ CAS Key Laboratory of Infection and Immunity, National Laboratory of Macromolecules, Institute of Biophysics, Chinese Academy of Sciences, Beijing, China. ${ }^{6}$ National Institute for Communicable Disease Control and Prevention, Chinese Center for Disease Control and Prevention, Changping, Beijing, China. IInstitute of Microbiology and Epidemiology, Academy of Military Medical Sciences, Beijing, China. *These authors contributed equally to this work.
\end{abstract}

†Corresponding author. Email: qinchuan@pumc.edu.cn (C.Q.); yjzhang@cdc.zj.cn (Y.Z.); yinwd@sinovac.com (W.Y.); xiangxi@ibp.ac.cn (X.W.); changguili@aliyun.com (C.L.); lujinxing@icdc.cn (J.L.)

The coronavirus disease 2019 (COVID-19) pandemic caused by severe acute respiratory syndromecoronavirus 2 (SARS-CoV-2) has resulted in an unprecedented public health crisis. There are currently no SARS-CoV-2-specific treatments or vaccines available due to the novelty of the virus. Hence, rapid development of effective vaccines against SARS-CoV-2 are urgently needed. Here we developed a pilotscale production of a purified inactivated SARS-CoV-2 virus vaccine candidate (PiCoVacc), which induced SARS-CoV-2-specific neutralizing antibodies in mice, rats and non-human primates. These antibodies neutralized 10 representative SARS-CoV-2 strains, suggesting a possible broader neutralizing ability against SARS-CoV-2 strains. Three immunizations using two different doses ( $3 \mu \mathrm{g}$ or $6 \mu \mathrm{g}$ per dose) provided partial or complete protection in macaques against SARS-CoV-2 challenge, respectively, without observable antibody-dependent enhancement of infection. These data support clinical development of SARS-CoV-2 vaccines for humans.

The World Health Organization declared the outbreak of coronavirus disease in 2019 (COVID-19) to be a Public Health Emergency of International Concern on 30 January 2020, and a pandemic on 11 March 2020. It is reported that $\sim 80 \%$ of COVID-19 patients have mild-to-moderate symptoms, while $\sim 20 \%$ develop serious manifestations such as severe pneumonia, acute respiratory distress syndrome (ARDS), sepsis and even death (1). The number of COVID-19 cases has increased at a staggering rate globally. Severe acute respiratory syndrome-coronavirus 2 (SARS-CoV-2), the causative virus of the ongoing pandemic, belongs to the genus Betacoronavirus ( $\beta-\mathrm{CoV})$ of the family Coronavirdae (2). SARS-CoV-2 along with the severe acute respiratory syndrome coronavirus (SARS-CoV) and the Middle Eastern respiratory syndrome-related coronavirus (MERS-CoV), constitute the three most life-threatening species among all human coronaviruses. SARS-CoV-2 harbors a linear singlestranded positive sense RNA genome, encoding 4 structural proteins [spike (S), envelope (E), membrane (M), and nucleocapsid $(\mathrm{N})$ ] of which $\mathrm{S}$ is a major protective antigen that elicits highly potent neutralizing antibodies (NAbs), 16 nonstructural proteins (nsp1-nsp16) and several accessory proteins (3). No specific antiviral drugs or vaccines against the newly emerged SARS-CoV-2 are currently available. Therefore, urgency in the development of vaccines is of vital importance to curb the pandemic and prevent new viral outbreaks.

Multiple SARS-CoV-2 vaccine types, such as DNA-, RNAbased formulations, recombinant-subunits containing viral epitopes, adenovirus-based vectors and purified inactivated virus are under development (4-6). Purified inactivated viruses have been traditionally used for vaccine development and such vaccines have been found to be safe and effective for the prevention of diseases caused by viruses like influenza virus and poliovirus $(7,8)$. To develop preclinical in vitro neutralization and challenge models for a candidate SARS-CoV2 vaccine, we isolated SARS-CoV-2 strains from the bronchoalveolar lavage fluid (BALF) samples of 11 hospitalized patients (including 5 patients in intensive care), among which 5 are from China, 3 from Italy, 1 from Switzerland, 1 from UK 
and 1 from Spain (table S1). These patients were infected with SARS-CoV-2 during the most recent outbreak. The 11 samples contained SARS-CoV-2 strains that are widely scattered on the phylogenic tree constructed from all available sequences, representing, to some extent, circulating SARS-CoV-2 populations (Fig. 1A and fig. S1). We chose strain $\mathrm{CN} 2$ for purified inactivated SARS-CoV-2 virus vaccine development (PiCoVacc) and another 10 strains (termed as $\mathrm{CN1}, \mathrm{CN} 3-\mathrm{CN} 5$ and OS1-OS6) as preclinical challenge strains. Of note, the $\mathrm{CN1}$ and OS1 strains are closely related to 2019-nCoV-BetaCoV /Wuhan/WIV04/2019 and EPI_ISL_412973, respectively, which have been reported to cause severe clinical symptoms, including respiratory failure, requiring mechanical ventilation $(9,10)$.

To obtain a viral stock adapted for efficient growth in Vero cells for PiCoVacc production, the CN2 strain was first plaque purified and passaged once in Vero cells to generate the P1 stock. After this another four passages were performed to generate the P2-P5 stocks. Growth kinetics analysis of the P5 stock in Vero cells showed that this stock replicated efficiently and reached a peak titer of $6-7 \log _{10} \mathrm{TCID}_{50} / \mathrm{ml}$ by 3 or 4 days post infection (dpi) at multiplicities of infection (MOI) of 0.0001-0.01 at temperatures between $33^{\circ} \mathrm{C}-37^{\circ} \mathrm{C}$ (Fig. 1B). To evaluate the genetic stability of PiCoVacc, 5 more passages were performed to obtain the P10 stock, whole genome of which, together with those of the P1, P3 and P5 stocks were sequenced. Compared to P1, only two amino acid substitutions, Ala $\rightarrow$ Asp at E residue 32 (E-A32D) and $\mathrm{Thr} \rightarrow$ Ile at nsp10 residue 49 (nsp10-T49I), occurred in P5 and P10 stocks (table S2), suggesting that PiCoVacc CN2 strain possesses excellent genetic stability without the $\mathrm{S}$ mutations that might potentially alter the NAb epitopes. To produce pilot scale PiCoVacc for animal studies, the virus was propagated in a 50-liter culture of Vero cells using the Cell Factory system and inactivated by using $\beta$-propiolactone (Fig. 1C). The virus was purified using depth filtration and two optimized steps of chromatography, yielding a highly pure preparation of PiCoVacc (Fig. 1D). Additionally, cryo-electron microscopy (cryoEM) analysis showed intact oval-shaped particles with diameters of 90-150 nm, which are embellished with crown-like spikes, representing a prefusion state of the virus (Fig. 1E).

To assess the immunogenicity of PiCoVacc, groups of $\mathrm{BALB} / \mathrm{c}$ mice $(\mathrm{n}=10)$ were injected at day 0 and day 7 with various doses of PiCoVacc mixed with alum adjuvant $(0,1.5$ or 3 or $6 \mu \mathrm{g}$ per dose, $0 \mu \mathrm{g}$ in physiological saline as the sham group). No inflammation or other adverse effects were observed. Spike-, receptor binding domain (RBD)-, and N-specific antibody responses were evaluated by enzyme-linked immunosorbent assays (ELISAs) at weeks 1-6 after initial immunization (fig. S2). SARS-CoV-2 S- and RBD-specific immunoglobulin G (Ig G) developed quickly in the serum of vaccinated mice and peaked at the titer of $819,200(>200$ $\mu \mathrm{g} / \mathrm{ml})$ and 409,600 (>100 $\mu \mathrm{g} / \mathrm{ml})$, respectively, at week 6 (Fig. 2A). RBD-specific IgG accounts for half of the S-induced antibody responses, suggesting RBD is the dominant immunogen, which closely matches the serological profile of the blood of recovered COVID-19 patients (Fig. 2, A and B) (11). Surprisingly, the amount of N-specific IgG induced is $\sim 30$ fold lower than the antibodies targeting S or RBD in immunized mice (Fig. 2A). Interestingly, previous studies have shown that the N-specific IgG is largely abundant in the serum of COVID-19 patients and serves as one of the clinical diagnostic markers (11). It's worthy to note that PiCoVacc could elicit 10-fold higher S-specific antibody titers in mice than those of the serum from the recovered COVID-19 patients (Fig. 2, A and B). Although this observation is currently not indicative of PiCoVacc's ability to produce similar results in humans, it highlights the potential of PiCoVacc to induce a strong and potent immune response. Taken together, our findings - coupled with the fact that the antibodies targeting $\mathrm{N}$ of SARS-CoV-2 do not provide protective immunity against the infection (12) - suggest that PiCoVacc might be capable of eliciting more effective antibody responses (Fig. 2, A and B).

Next, we measured SARS-CoV-2-specific neutralizing antibodies over a period of time using microneutralization assays (MN50). Similar to S-specific IgG responses, the neutralizing antibody titer against the $\mathrm{CN} 1$ strain emerged at week 1 (12 for high dose immunization), surged after the week 2 booster and reached up to around 1,500 for low and medium doses, and 3,000 for the high dose at week 7, respectively (Fig. 2A). In contrast, the sham group did not develop detectable SARS-CoV-2-specific antibody responses (Fig. 2, A and $\mathrm{B})$. In addition, immunogenic evaluations of PiCoVacc in Wistar rats with the same immunization strategy yielded similar results - the maximum neutralizing titers reached 2,0484,096 at week 7 (Fig. 2C). To investigate the spectrum of neutralizing activities elicited by PiCoVacc, we conducted neutralization assays against the other 9 isolated SARS-CoV-2 strains using mouse and rat serums collected 3 weeks post vaccination. Neutralizing titers against these strains demonstrate that PiCoVacc is capable of eliciting antibodies that possibly exhibit potent neutralization activities against SARSCov-2 strains circulating worldwide (Fig. 2, D and E).

We next evaluated the immunogenicity and protective efficacy of PiCoVacc in rhesus macaques (Macaca mulatta), a non-human primate species that shows a COVID-19-like disease caused by SARS-CoV-2 infection (13). Macaques were immunized three times via the intramuscular route with medium ( $3 \mu \mathrm{g}$ per dose) or high doses ( $6 \mu \mathrm{g}$ per dose) of PiCoVacc at day 0, 7 and $14(n=4)$. S-specific IgG and NAb were induced at week 2 and rose to $\sim 12,800$ and $\sim 50$, respectively at week 3 (before virus challenge) in both vaccinated groups, whose titers are similar to those of serum from the recovered COVID-19 patients (Fig. 3, A and B). Unexpectedly, NAb titer 
(61) in the medium dose immunized group were $\sim 20 \%$ greater than that observed (50) in the high dose vaccinated group at week 3, possibly due to individual differences in the ability of one animal in the medium dose group in eliciting $\sim 10$-fold higher titer when compared to the other three animals (Fig. 3B). Excluding this exception, NAb titer in the medium dose group would drop down to $34, \sim 40 \%$ lower than that in the high dose group. Subsequently, we conducted a challenge study by a direct inoculation of $10^{6} \mathrm{TCID}_{50}$ of SARSCoV-2 CN1 into the animal lung through the intratracheal route at day 22 (one week after the third immunization) in vaccinated and control macaques to verify the protective efficacy. Expectedly, all control (sham and placebo) macaques showed excessive copies $\left(10^{4}-10^{6} / \mathrm{ml}\right)$ of viral genomic RNA in the pharynx, crissum and lung by day 3-7 post-inoculation (dpi) and severe interstitial pneumonia (Fig. 3, C to F). By contrast, all vaccinated macaques were largely protected against SARS-CoV-2 infection with very mild and focal histopathological changes in a few lobes of lung, which probably were caused by a direct inoculation of $10^{6} \mathrm{TCID}_{50}$ of virus into the lung through intratracheal route, that needed longer time (more than one week) to recover completely (Fig. 3F). Viral loads decreased significantly in all vaccinated macaques, but increased slightly in control animals from day 3-7 after infection (Fig. 3, C to E). All four macaques that received the high dose, had no detectable viral loads in pharynx, crissum and lung at day 7 after infection. In the medium dose immunized group, we indeed partially detected the viral blip from pharyngeal (3/4), anal (2/4) and pulmonary (1/4) specimens at day 7 after infection, while viral loads presented a $\sim 95 \%$ reduction when compared to the sham groups (Fig. 3, C to E). Interestingly, NAb titer in vaccinated groups decreased by $\sim 30 \%$ by 3 days post infection to neutralize viruses, then rapidly increased from day 5-7 after infection to maintain neutralization efficacy. In comparison with high dose vaccination group (titer of $\sim 145$ ), higher NAb titers observed in the medium dose vaccinated group at day 7 after infection $(\sim 400$ for 4 macaques) might have resulted from relatively low levels of viral replication, suggesting a requirement of longer time for complete viral clearance. No antibody-dependent enhancement (ADE) of infection was observed for the vaccinated macaques despite the observation that relatively low NAb titer existed within the medium dose group before infection, offering partial protection. The possibility of manifestation of ADE after antibody titers wane could not be ruled out in this study. Further studies involving observation of challenged animals at longer periods of time post vaccination are warranted to address this.

Previous reports on the development of SARS and MERS vaccine candidates raised concerns about pulmonary immunopathology, either directly caused by a type 2 helper Tcell (Th2) response or as a result of $\mathrm{ADE}(4,14,15)$. Although
T-cell responses elicited by many vaccines have been demonstrated to be crucial for acute viral clearance, protection from subsequent coronavirus infections is largely mediated by humoral immunity $(16,17)$. The "cytokine storm" induced by excessive T-cell responses have been actually shown to accentuate the pathogenesis of COVID19 $(18,19)$. Therefore, T-cell responses elicited by any SARS-CoV-2 vaccine(s) would have to be well controlled in order to avoid immunopathology. In this context, we systematically evaluated safety of PiCoVacc in macaques by recording a number of clinical observations and biological indices. Two groups of macaques $(n=10)$ were immunized by intramuscular injection with low $(1.5 \mu \mathrm{g})$ or high doses $(6 \mu \mathrm{g})$ and another two groups of macaques $(n=10)$ were immunized with adjuvant (sham) and physiological saline (placebo) for three times at day 0,7 and 14 time points. Neither fever nor weight loss was observed in any macaque after the immunization of PiCoVacc, and the appetite and mental state of all animals remained normal (fig. S3). Hematological and biochemical analysis, including biochemical blood test, lymphocyte subset percent $\left(\mathrm{CD}^{+}\right.$, $\mathrm{CD}^{+}$and $\mathrm{CD}^{+}$) and key cytokines (TNF- $\alpha$, IFN- $\gamma$, IL-2, IL-4, IL-5 and IL-6) showed no notable changes in vaccinated groups when compared to the sham and placebo groups (Fig. 4, A and B, and figs. S4 and S5). In addition, histopathological evaluations of various organs, including lung, heart, spleen, liver, kidney and brain, from the 4 groups at day 29 demonstrated that PiCoVacc did not cause any notable pathology in macaques (Fig. 4C and fig. S6).

The serious pandemic of the current COVID19 and the precipitously increasing numbers of death worldwide necessitate the urgent development of a SARS-CoV-2 vaccine, requiring a new pandemic paradigm. The safety and efficacy are essential for vaccine development at both stages of preclinical studies and clinical trials. Although it's still too early to define the best animal model for studying SARS-CoV-2 infections, rhesus macaques that mimic COVID-19-like symptoms after SARS-CoV-2 infection appear promising animal models for studying the disease. We provide evidences for the safety of PiCoVacc in macaques; and did not observe infection enhancement or immunopathological exacerbation in our studies. Our data also demonstrate a complete protection against SARS-CoV-2 challenge with a $6 \mu \mathrm{g}$ per dose of PiCoVacc in macaques. Collectively these results suggest a path forward for clinical development of SARS-CoV-2 vaccines for use in humans. Phases I, II and III clinical trials with PiCoVacc, as well as other SARS-CoV-2 vaccine candidates, are expected to begin later this year.

\section{REFERENCES AND NOTES}

1. Z. Wu, J. M. McGoogan, Characteristics of and important lessons from the coronavirus disease 2019 (COVID-19) outbreak in China: Summary of a report of 72,314 cases from the Chinese Center for Disease Control and Prevention. JAMA 323, 1239-1242 (2020). doi:10.1001/jama.2020.2648 Medline

2. Coronaviridae Study Group of the International Committee on Taxonomy of 
Viruses, The species severe acute respiratory syndrome-related coronavirus: Classifying 2019-nCoV and naming it SARS-CoV-2. Nat. Microbiol. 5, 536-544 (2020). doi:10.1038/s41564-020-0695-z Medline

3. A. Wu, Y. Peng, B. Huang, X. Ding, X. Wang, P. Niu, J. Meng, Z. Zhu, Z. Zhang, J. Wang, J. Sheng, L. Quan, Z. Xia, W. Tan, G. Cheng, T. Jiang, Genome composition and divergence of the novel coronavirus (2019-nCoV) originating in China. Cell Host Microbe 27, 325-328 (2020). doi:10.1016/i.chom.2020.02.001 Medline

4. N. Lurie, M. Saville, R. Hatchett, J. Halton, Developing Covid-19 vaccines at pandemic speed. N. Engl. J. Med. 10.1056/NEJMp2005630 (2020). doi:10.1056/NEJMp2005630 Medline

5. K. Dhama, K. Sharun, R. Tiwari, M. Dadar, Y. S. Malik, K. P. Singh, W. Chaicumpa, COVID-19, an emerging coronavirus infection: Advances and prospects in designing and developing vaccines, immunotherapeutics, and therapeutics. Hum. Vaccin. Immunother. 10.1080/21645515.2020.1735227 (2020). doi: $10.1080 / 21645515.2020 .1735227$ Medline

6. E. Kim, G. Erdos, S. Huang, T. W. Kenniston, S. C. Balmert, C. D. Carey, V. S. Raj, M. W. Epperly, W. B. Klimstra, B. L. Haagmans, E. Korkmaz, L. D. Falo Jr., A. Gambotto, Microneedle array delivered recombinant coronavirus vaccines: Immunogenicity and rapid translational development. EBioMedicine 102743 (2020). doi:10.1016/i.ebiom.2020.102743 Medline

7. A. D. Murdin, L. Barreto, S. Plotkin, Inactivated poliovirus vaccine: Past and present experience. Vaccine 14, 735-746 (1996). doi:10.1016/0264-410X(95)00211-1 Medline

8. C. Vellozzi, D. R. Burwen, A. Dobardzic, R. Ball, K. Walton, P. Haber, Safety of trivalent inactivated influenza vaccines in adults: Background for pandemic influenza vaccine safety monitoring. Vaccine $27,2114-2120$ (2009). doi:10.1016/i vaccine.2009.01.125 Medline

9. P. Stefanelli, G. Faggioni, A. Lo Presti, S. Fiore, A. Marchi, E. Benedetti, C. Fabiani, A. Anselmo, A. Ciammaruconi, A. Fortunato, R. De Santis, S. Fillo, M. R. Capobianchi, M. R. Gismondo, A. Ciervo, G. Rezza, M. R. Castrucci, F. Lista; On Behalf of Iss Covid-Study Group, Whole genome and phylogenetic analysis of two SARS-CoV-2 strains isolated in Italy in January and February 2020: Additional clues on multiple introductions and further circulation in Europe. Euro. Surveill. 25, 2000305 (2020). doi:10.2807/1560-7917.ES.2020.25.13.2000305 Medline

10. P. Zhou, X.-L. Yang, X.-G. Wang, B. Hu, L. Zhang, W. Zhang, H.-R. Si, Y. Zhu, B. Li, C.-L. Huang, H.-D. Chen, J. Chen, Y. Luo, H. Guo, R.-D. Jiang, M.-Q. Liu, Y. Chen, X.-R. Shen, X. Wang, X.-S. Zheng, K. Zhao, Q.-J. Chen, F. Deng, L.-L. Liu, B. Yan, F.X. Zhan, Y.-Y. Wang, G.-F. Xiao, Z.-L. Shi, A pneumonia outbreak associated with a new coronavirus of probable bat origin. Nature 579, 270-273 (2020). doi:10.1038/s41586-020-2012-7 Medline

11. L. Liu, S. Wang, S. Zheng, A preliminary study on serological assay for severe acute respiratory syndrome coronavirus 2 (SARS-CoV-2) in 238 admitted hospital patients. MedRxiv 2020.03.06.20031856 [Preprint]. 8 March 2020; https://doi.org/10.1101/2020.03.06.20031856.

12. L. E. Gralinski, V. D. Menachery, Return of the coronavirus: 2019-nCoV. Viruses 12 , E135 (2020). doi:10.3390/v12020135 Medline

13. P. Yu, F. Qi, Y. Xu, F. Li, P. Liu, J. Liu, L. Bao, W. Deng, H. Gao, Z. Xiang, C. Xiao, Q. Lv, S. Gong, J. Liu, Z. Song, Y. Qu, J. Xue, Q. Wei, M. Liu, G. Wang, S. Wang, H. Yu, X. Liu, B. Huang, W. Wang, L. Zhao, H. Wang, F. Ye, W. Zhou, W. Zhen, J. Han, G. Wu, Q. Jin, J. Wang, W. Tan, C. Qin, Age-related rhesus macaque models of COVID19. Animal Model. Exp. Med. 3, 93-97 (2020). doi:10.1002/ame2.12108 Medline

14. C. T. Tseng, E. Sbrana, N. Iwata-Yoshikawa, P. C. Newman, T. Garron, R. L. Atmar, C. J. Peters, R. B. Couch, Correction: Immunization with SARS coronavirus vaccines leads to pulmonary immunopathology on challenge with the SARS virus. PLOS ONE 7, e35421 (2012). doi:10.1371/annotation/2965cfae-b77d-4014-8b7b236e01a35492 Medline

15. C. Y. Yong, H. K. Ong, S. K. Yeap, K. L. Ho, W. S. Tan, Recent advances in the vaccine development against Middle East respiratory syndrome-coronavirus. Front. Microbiol. 10, 1781 (2019). doi:10.3389/fmicb.2019.01781 Medline

16. E. Prompetchara, C. Ketloy, T. Palaga, Immune responses in COVID-19 and potential vaccines: Lessons learned from SARS and MERS epidemic. Asian Pac. J. Allergy Immunol. 38, 1-9 (2020). Medline

17. J. Zhao, R. A. P. M. Perera, G. Kayali, D. Meyerholz, S. Perlman, M. Peiris, Passive immunotherapy with dromedary immune serum in an experimental animal model for Middle East respiratory syndrome coronavirus infection. J. Virol. 89, 6117-
6120 (2015). doi:10.1128/JVI.00446-15 Medline

18. J. M. Nicholls, L. L. M. Poon, K. C. Lee, W. F. Ng, S. T. Lai, C. Y. Leung, C. M. Chu, P. K. Hui, K. L. Mak, W. Lim, K. W. Yan, K. H. Chan, N. C. Tsang, Y. Guan, K. Y. Yuen, J. S. Peiris, Lung pathology of fatal severe acute respiratory syndrome. Lancet 361, 1773-1778 (2003). doi:10.1016/S0140-6736(03)13413-7 Medline

19. H. Y. Zheng, M. Zhang, C. X. Yang, N. Zhang, X. C. Wang, X. P. Yang, X. Q. Dong, Y. $T$. Zheng, Elevated exhaustion levels and reduced functional diversity of $T$ cells in peripheral blood may predict severe progression in COVID-19 patients. Cell. Mol. Immunol. 17, 541-543 (2020). doi:10.1038/s41423-020-0401-3 Medline

20. J. Miller, R. Ulrich, On the analysis of psychometric functions: The SpearmanKärber method. Percept. Psychophys. 63, 1399-1420 (2001). doi:10.3758/BF03194551 Medline

21. L. T. Nguyen, H. A. Schmidt, A. von Haeseler, B. Q. Minh, IQ-TREE: A fast and effective stochastic algorithm for estimating maximum-likelihood phylogenies. Mol. Biol. Evol. 32, 268-274 (2015). doi:10.1093/molbev/msu300 Medline

22. G. C. Yu, D. K. Smith, H. C. Zhu, Y. Guan, T. T. Y. Lam, ggtree: An r package for visualization and annotation of phylogenetic trees with their covariates and other associated data. Methods Ecol. Evol. 8, 28-36 (2017). doi:10.1111/2041210X.12628

23. N. Wang, D. Zhao, J. Wang, Y. Zhang, M. Wang, Y. Gao, F. Li, J. Wang, Z. Bu, Z. Rao, $X$. Wang, Architecture of African swine fever virus and implications for viral assembly. Science 366, 640-644 (2019). doi:10.1126/science.aaz1439 Medline

\section{ACKNOWLEDGMENTS}

We thank F. Gao, Z. Rao and J. Wang for project discussion, W. Zhai and M. Li for phylogenetic analysis. We gratefully acknowledge the researchers that submitted sequences to the GISAID's EpiFlu Database on which this research is based. Oiang Gao, Lin Wang, Yajing Li, Zhe Lv, Xiaogin Ge, Fang Cai, Yanhui Yin and Yurong Li are inventors on patent application (PCT/CN2020/086892 and PCT/CN2020/085413) held/submitted by Sinovac Biotech Ltd that covers "inactivated vaccine for SARS-CoV-2 and the preparation thereof". Research was supported by the National Key Research and Development Program (2020YFC0842100, 2020YFA0707500), the Strategic Priority Research Program (XDB29010000), the CAMS initiative for Innovative Medicine of China (Grant No. 2016-I2M-2-006), National Mega projects of China for Major Infectious Diseases (2017ZX10304402), National Key Research and Development Project of China (Grant No. 2016YFD0500304), Zhejiang Province Science and Technology Major Project (2020C03124) and the Beijing science and technology plan (Z201100005420006). X. W was supported by Ten Thousand Talent Program and the NSFS Innovative Research Group (No. 81921005). Author contributions: L.B., H.M., L.W., K. Xu., Y-J. L., H.G., X.G., B.K., Y.H., J.L., F.C., D.J., Y.Y., C-F.Q., J.L., X.L., W.S., D.W., H.Z., L.Z., W.D., and YR.L. performed experiments; Q.G., C.Q., Y.Z.,W.Y., X.W., C.L., J-X.L. and X.G. designed the study; all authors analyzed data; and X.W. wrote the manuscript. Competing interests: Q.G., L.W., Y.L., Z.L., X.G., F.C., Y.Y., and Y.L. are inventors on patent application (PCT/CN2020/086892 and PCT/CN2020/085413) submitted by Sinovac Biotech Ltd that covers "inactivated vaccine for SARSCoV-2 and the preparation thereof". Data and materials availability: The complete genome sequences of SARS-CoV-2 used in this study have been deposited in GenBank under accession number MT407649, MT407650, MT407651, MT407652, MT407653, MT407654, MT407655, MT407656, MT407657, MT407658 and MT407659, respectively. This work is licensed under a Creative Commons Attribution 4.0 International (CC BY 4.0) license, which permits unrestricted use, distribution, and reproduction in any medium, provided the original work is properly cited. To view a copy of this license, visit https:/creativecommons.org/licenses/by/4.0/. This license does not apply to figures/photos/artwork or other content included in the article that is credited to a third party; obtain authorization from the rights holder before using such material. 


\section{SUPPLEMENTARY MATERIALS}

science. sciencemag.org/cgi/content/full/science.abc1932/DC1

Materials and Methods

Figs. S1 to S6

Tables S1 and S2

References (20-23)

10 April 2020; accepted 2 May 2020

Published online 6 May 2020

$10.1126 /$ science.abc1932 


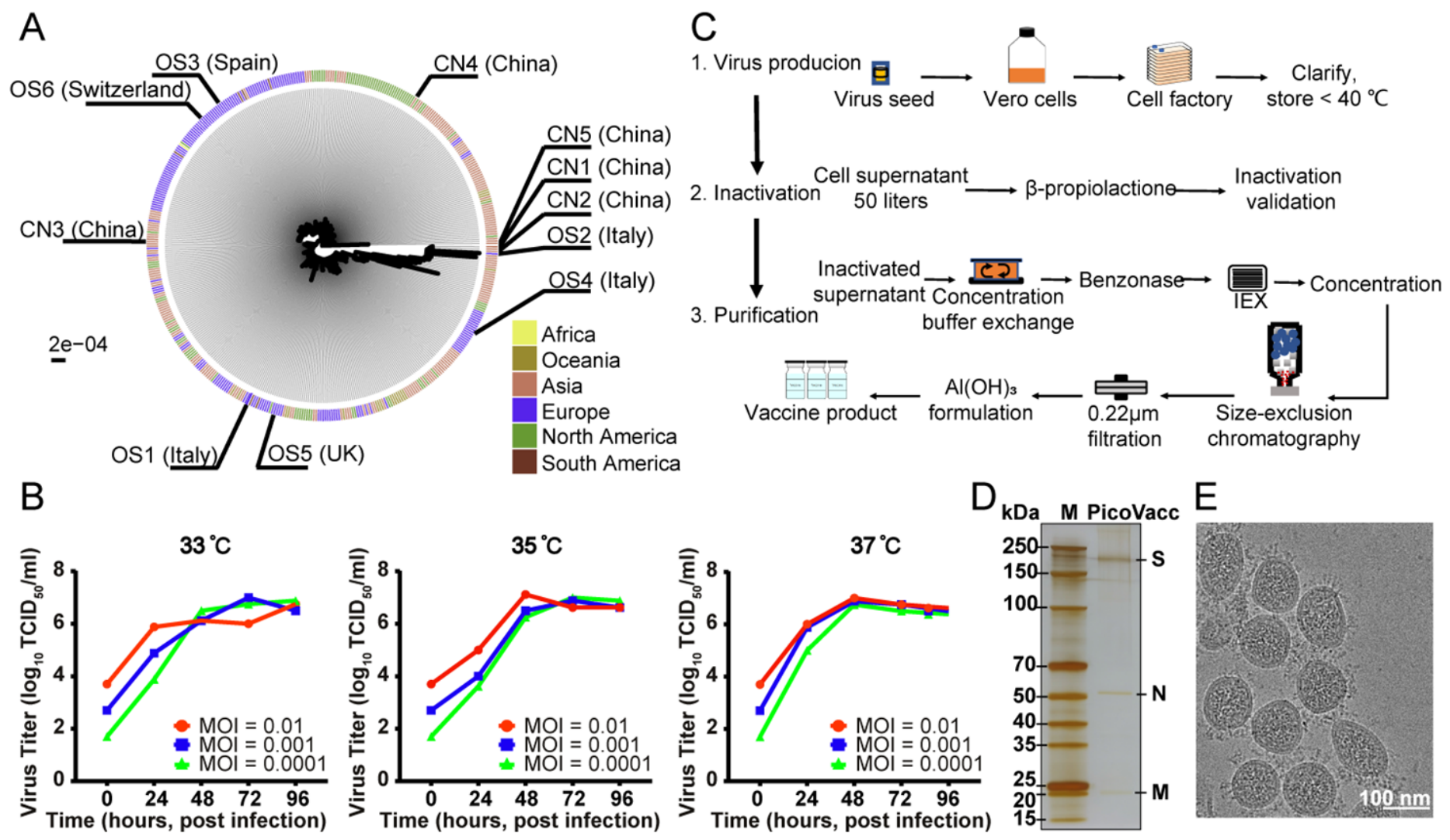

Fig. 1. Characterization of the SARS-CoV-2 vaccine candidate, PiCoVacc. (A) The SARS-CoV-2 maximum likelihood phylogenetic tree. The SARS-CoV-2 isolates used in this study are depicted with black lines and labeled. Viral strains were isolated in infected patients who traveled from the continents as indicated (B) Growth kinetics of PiCoVacc (CN2) P5 stock in Vero cells. (C) Flowchart of PiCoVacc preparation. (D) Protein composition and purity evaluation of PiCoVacc by NuPAGE 4-12\% Bis-Tris Gel. (E) Representative electron micrograph of PiCoVacc. White scale bar $=100 \mathrm{~nm}$. 
A

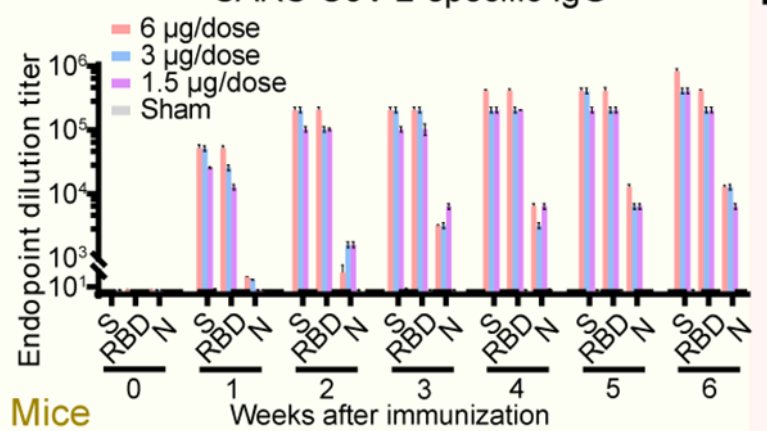

Mice

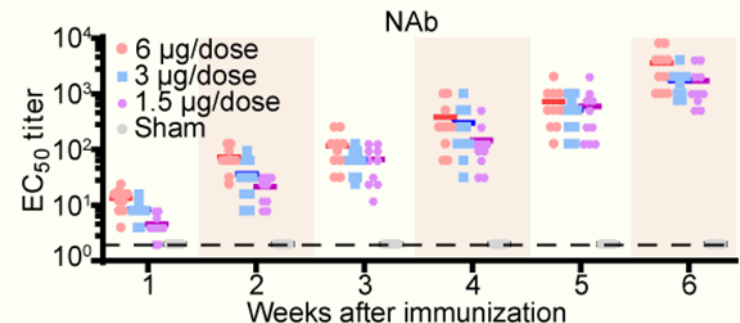

B

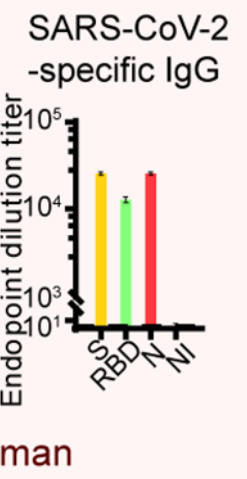

C

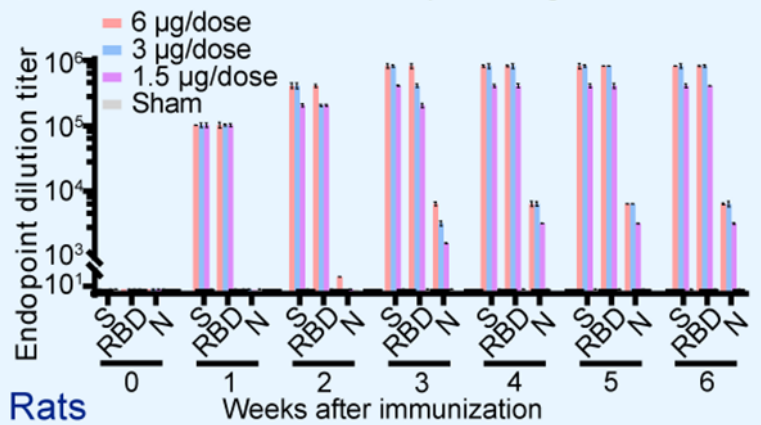

Rats

Weeks after immunization

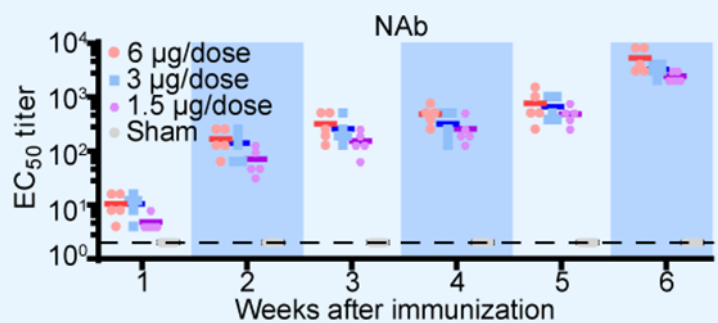

D

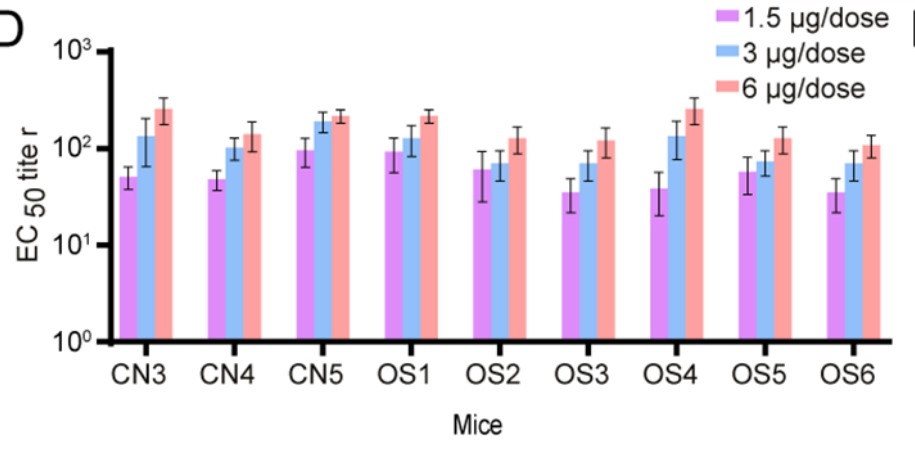

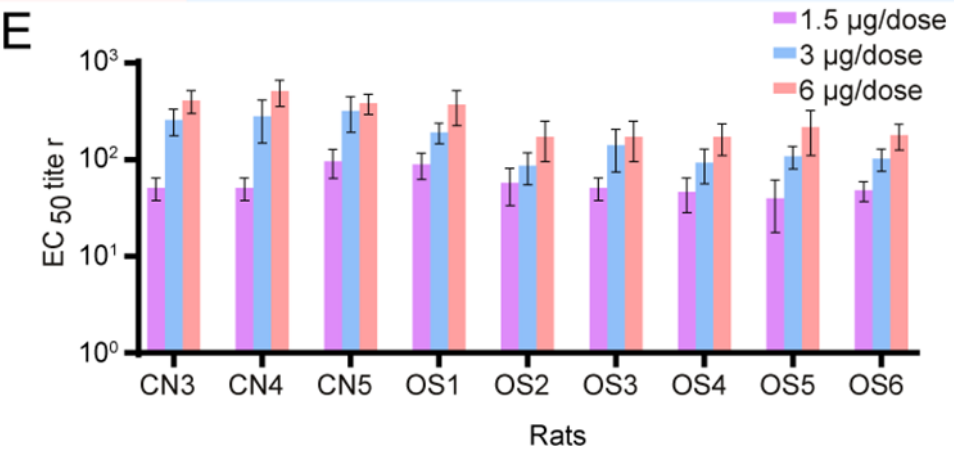

Fig. 2. PiCoVacc immunization elicits neutralizing antibody response against ten representative SARS-CoV-2 isolates. BALB/c mice and Wistar rats were immunized with various doses of PiCoVacc or control (adjuvant only as the sham group) $(n=10)$. Serums from recovered COVID19 patients (RECOV) and non-infected (NI) individuals were used as positive and negative controls, respectively. The antibody responses were analyzed in mice (A), humans (B) and rats (C). Top: SARS-CoV-2-specific IgG responses as measured by ELISA; bottom: neutralizing antibody titer determined by microneutralization assay. The spectrum of neutralizing activities elicited by PiCoVacc was investigated in mice (D) and rats (E). Neutralization assays against the other nine isolated SARS-CoV-2 strains was performed using mouse and rat serums collected 3 weeks post-vaccination. Data points represent mean $+/-$ SEM of individual animals and humans from five to ten independent experiments; error bars reflect SEM;; dotted lines indicate the limit of detection; horizontal lines indicate the geometric mean titer (GMT) of $\mathrm{EC}_{50}$ for each group. 

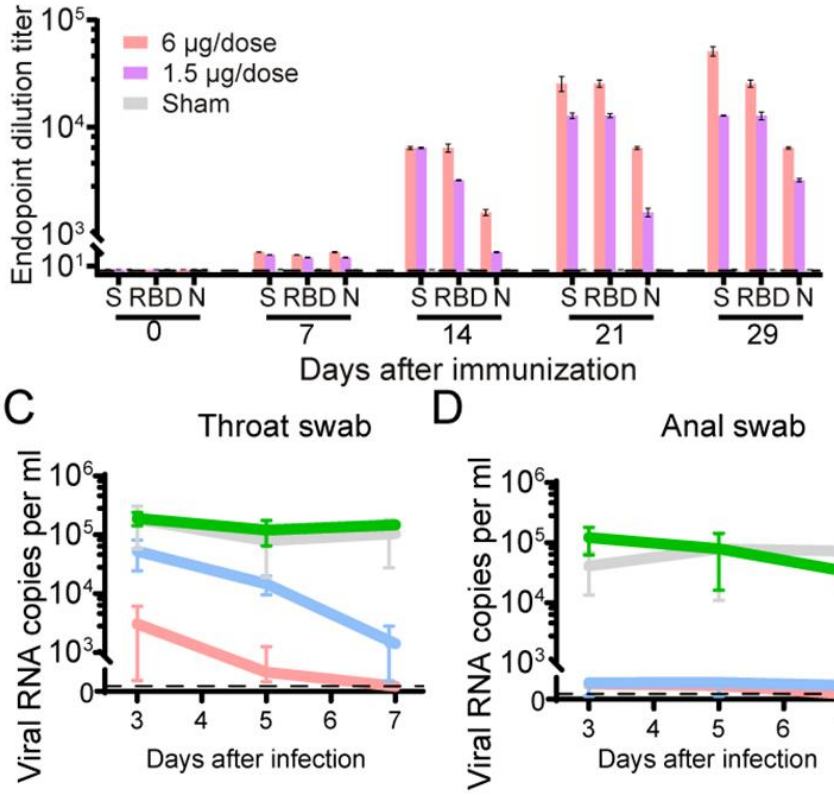

F

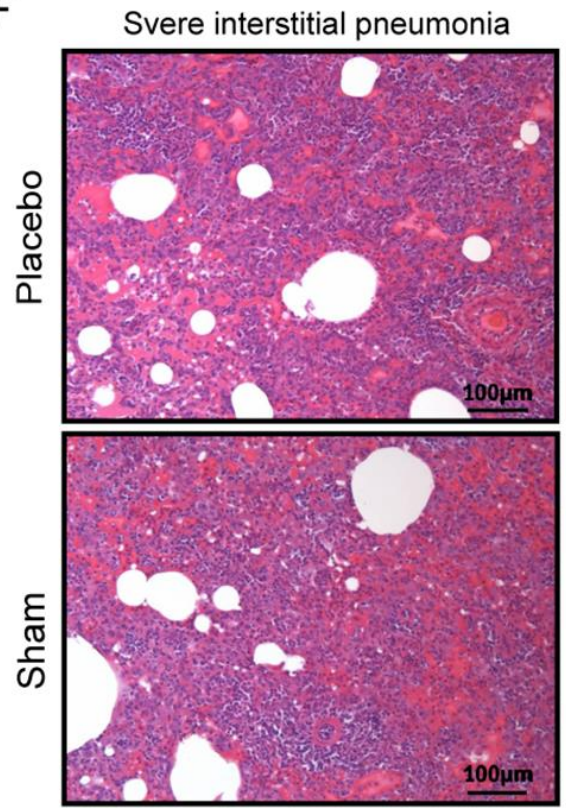

H.E. $\times 100$
Anal swab

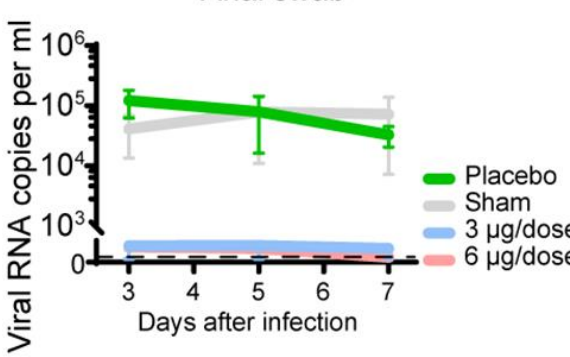

Mild interstitial pneumonia

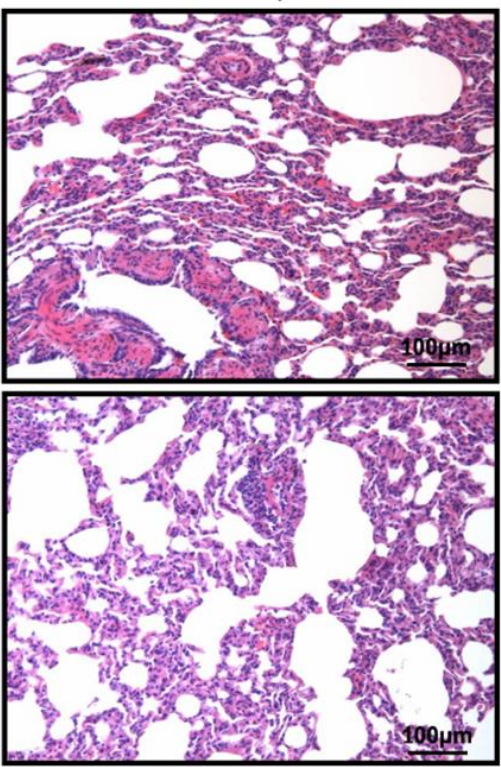

H.E. $\times 100$

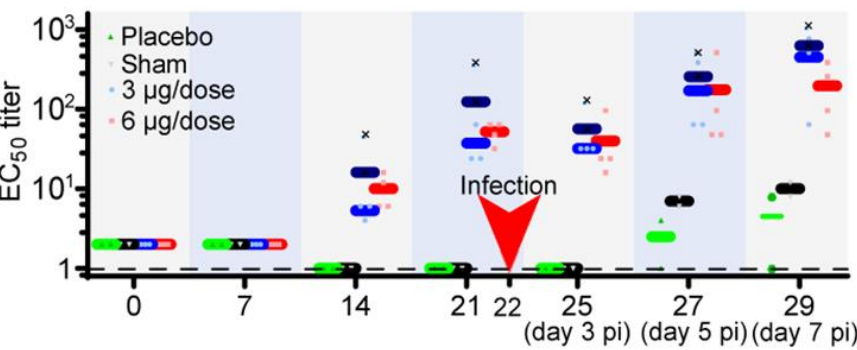

Days after vaccination

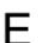

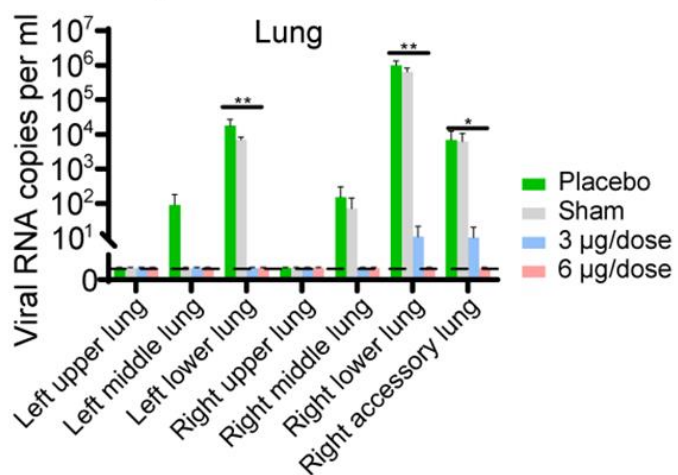

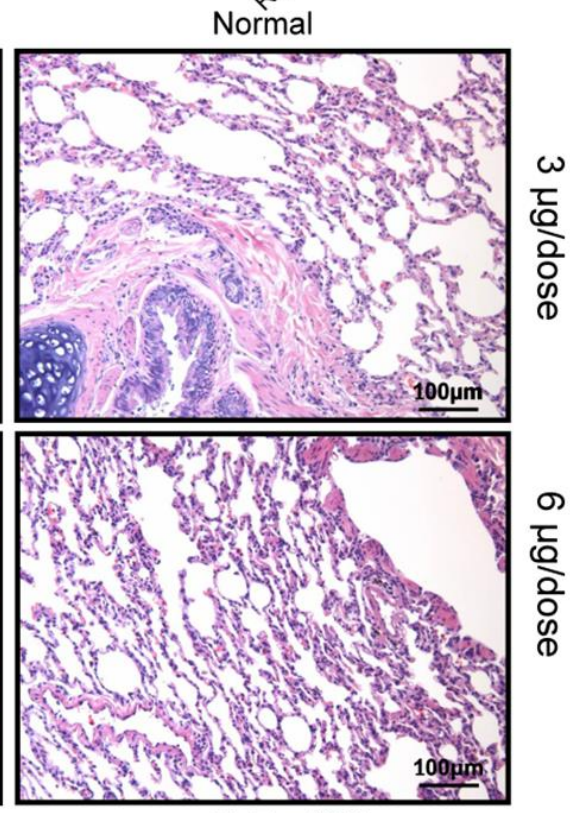

H.E. $\times 100$

Fig. 3. Immunogenicity and protective efficacy of PiCoVacc in nonhuman primates. Macaques were immunized three times through the intramuscular route with various doses of PiCoVacc or adjuvant only (sham) or placebo $(n=4)$. SARS-CoV-2-specific IgG response $(A)$ and neutralizing antibody titer (B) were measured. Data points represent mean +/- SEM of individual macaques from four independent experiments; error bars reflect SEM; dotted lines indicate the limit of detection; horizontal lines indicate the geometric mean titer (GMT) of EC 50 for each group. Protective efficacy of PiCoVacc against SARS-CoV-2 challenge at week 3 after immunization was evaluated in macaques (C-F). Viral loads of throat (C) and anal (D) swab specimens collected from the inoculated macaques at day 3, 5 and 7 pi were monitored. Viral loads in various lobes of lung tissue from all the inoculated macaques at day 7 post-infection were measured (E). RNA was extracted and viral load was determined by qRT-PCR. All data are presented as mean \pm SEM from four independent experiments; error bars reflect SEM. Asterisks represent significance: ${ }^{*} P<0.05$ and ${ }^{*} P<0.01$. Histopathological examinations $(F)$ in lungs from all the inoculated macaques at day 7 post infection. Lung tissue was collected and stained with hematoxylin and eosin. 


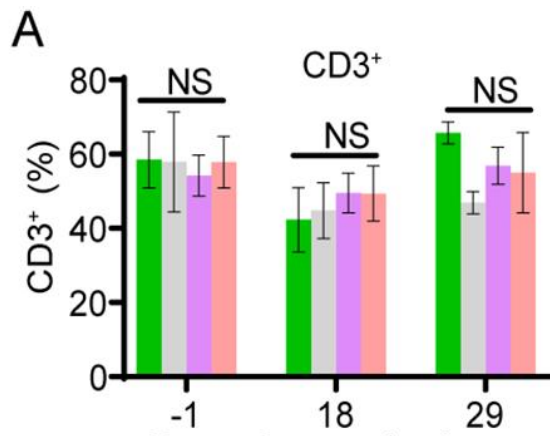

Days after vaccination

B

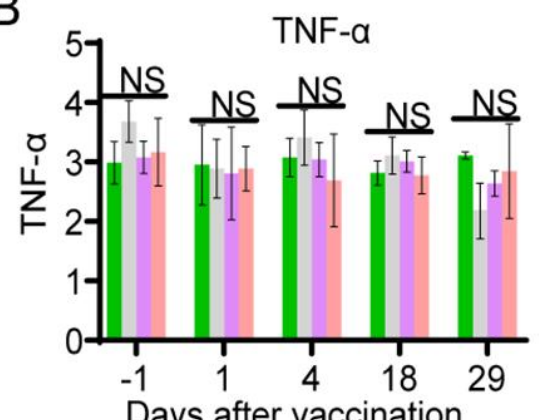

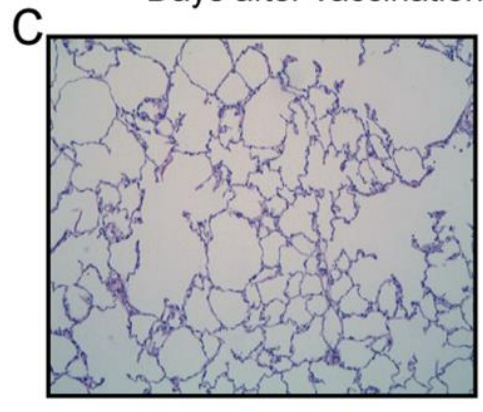

Placebo
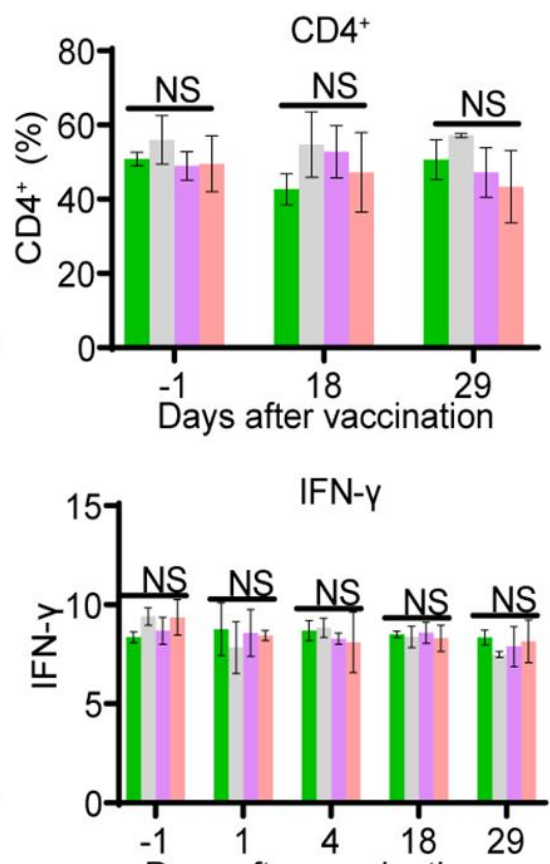

Days after vaccination
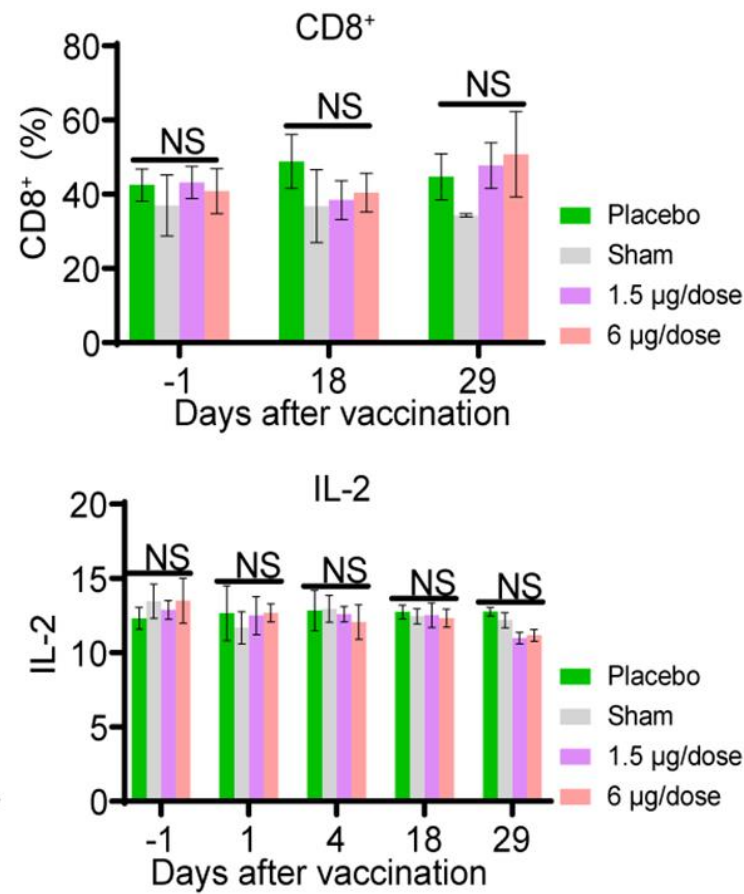

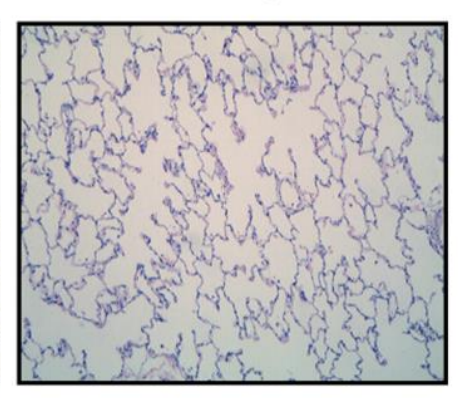

Sham

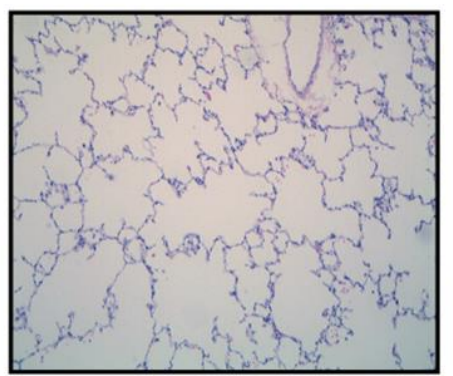

$1.5 \mu \mathrm{g} / \mathrm{dose}$

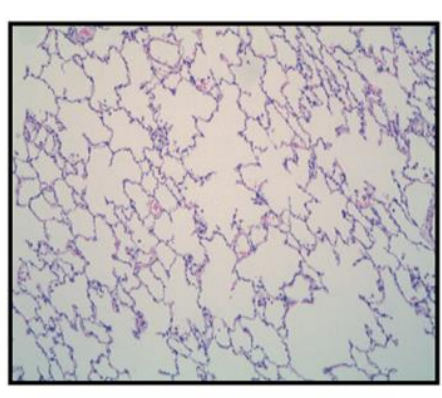

$6 \mu \mathrm{g} / \mathrm{dose}$

Fig. 4. Safety evaluation of PiCoVacc in nonhuman primates. Macaques were immunized three times at day 0, 7 and 14 through the intramuscular route with low dose $(1.5 \mu \mathrm{g}$ per dose) or high dose $(6 \mu \mathrm{g}$ per dose) of PiCoVacc or adjuvant only (sham) or placebo. (A and B) Hematological analysis in all four groups of macaques $(n=4)$. Lymphocyte subset percents (A), including $\mathrm{CD}^{+}, \mathrm{CD}^{+}$and $\mathrm{CD}^{+}$were monitored at day -1 (1 day before vaccination), 18 ( 3 days after the second vaccination) and 29 (7 days after the third vaccination). Key cytokines (B), containing TNF- $\alpha$, IFN- $\gamma$ and IL-2 were examined at day $-1,1$ (the day of the first vaccination), 4, 18 and 29 after vaccination. Data points show mean \pm SD from four independent experiments; error bars reflect SD. (C) Histopathological evaluations in lungs from four groups of macaques at day 29. Lung tissue was collected and stained with hematoxylin and eosin. 